\title{
QUALIDADE DE VIDA DE IDOSOS USUÁRIOS DE BENZODIAZEPÍNICOS
}

\author{
QUALITY OF LIFE OF ELDERLY BENZODIAZEPINIC USERS
}

\section{Sabrinna Maia Teixeira Soutoa, Márcia Helena Miranda Cardoso Podestáb, Walnéia Aparecida de Souzac', Grazieli Gianotti de Almeida ${ }^{\mathrm{d}}$}

\author{
asabrinna.farma@hotmail.com, bmhmcardoso@hotmail.com, cwalne23@yahoo.com.br, dgra_gianotti@yahoo.com.br \\ Universidade Federal de Alfenas - Alfenas (MG), Brasil
}

Data de recebimento do artigo: 20/02/2017 Data de aceite do artigo: 08/05/2017

\section{RESUMO}

Introdução: A prevalência de prescrição de benzodiazepínicos para idosos é elevada, porém, essa classe de medicamentos é potencialmente inadequada, devido a possíveis reaçốs adversas. As alteraçóes decorrentes do envelhecimento podem interferir na farmacocinética e farmacodinâmica dos medicamentos. Benzodiazepínicos de meia-vida longa, como o Diazepam, têm maior chance de envolver idosos em acidentes graves. Objetivo: Este estudo tem como objetivo avaliar a qualidade de vida de idosos atendidos pelo SUS na cidade de Alfenas que fazem uso contínuo de benzodiazepínico. Materiais e métodos: Foi realizado um estudo transversal com 100 idosos, utilizando um instrumento genérico de avaliaçáo da qualidade de vida, SF-36 (Medical Outcomes Study 36 - Item Short-Form Health Survey). A coleta de dados foi realizada entre junho e setembro de 2016, na CDM do município de Alfenas, por meio de entrevistas realizadas no momento da dispensação do benzodiazepínico. Resultados: A análise estatística não mostrou correlação significativa entre as médias, demonstrando, dessa forma, que não houve alteração da qualidade de vida dos usuários de Diazepam, quando comparados com outros benzodiazepínicos. Observou-se que $71(71 \%)$ dos pacientes eram do sexo feminino. O benzodiazepínico mais utilizado foi o Clonazepam (68\%), seguido do Diazepam (26\%), Lorazepam (4\%) e Bromazepam (2\%), respectivamente. Quanto à QV, a dimensão do SF-36 mais comprometida foi o aspecto físico $(44,6)$. O melhor índice foi encontrado na dimensão aspectos sociais $(63,9)$. Conclusóes: Os achados deste estudo evidenciam que há um comprometimento geral na qualidade de vida dos idosos usuários de benzodiazepínicos, residentes no município de Alfenas, Minas Gerais.

Palavras-chave: Benzodiazepínicos; idosos; qualidade de vida.

\section{ABSTRACT}

Introduction: The prevalence of benzodiazepine prescription for the elderly is high, but this class of drugs is potentially inappropriate due to possible adverse reactions. Changes due to aging may interfere with the pharmacokinetics and pharmacodynamics of medicinal products. Long-acting benzodiazepines like Diazepam are more likely to involve older people in serious accidents. Objective: The objective of this study was to evaluate the quality of life of the elderly who attended the SUS in the city of Alfenas, who use continuous benzodiazepine. Materials and methods: A cross-sectional study was carried out with 100 elderly patients using a generic instrument of quality of life evaluation, SF-36 (Item Short-Form Health Survey). Data collection was performed between June and September 2016, at the Medicinal Distribution Center (CDM) in the city of Alfenas, through interviews conducted at the time of the benzodiazepine dispensation. Results: The statistical analysis showed no significant correlation between the means, thus demonstrating that there was no change in the quality of life of Diazepam users when compared with other benzodiazepines. It was observed that 71 (71\%) of the patients were female. The most commonly used benzodiazepine was Clonazepam (68\%), followed by Diazepam (26\%), Lorazepam (4\%) and Bromazepam (2\%), respectively. Regarding QOL, the most compromised SF-36 was the physical aspect (44.6). The best index was found in the social aspects dimension (63.9). Conclusions: The findings of this study show that there is a general compromise in the quality of life of elderly benzodiazepine users, living in the city of Alfenas, Minas Gerais.

Keywords: Benzodiazepines; elderly; life quality. 


\section{Introdução}

Nas últimas décadas, tem-se observado crescimento significativo da população na faixa etária acima de 60 anos. Esse aumento do número de idosos gera maior consumo de medicamentos e maior busca por serviços de saúde. A alta prevalência de doenças crônicas e morbidades nesses pacientes apontam estes como principais usuários de medicamentos na sociedade $^{1}$. No processo de envelhecimento ocorrem alteraçôes fisiológicas que acarretam modificações na farmacocinética e farmacodinâmica de medicamentos, provocando uma série de mudanças na absorção do fármaco, distribuição, biotransformação e eliminação, além do declínio na manutenção da homeostase, com potenciais riscos à saúde. Além disso, a polifarmácia predispóe esses pacientes a um número maior de interaçôes medicamentosas, alimentares, dificuldades na adesão ao tratamento e reaçôes adversas a medicamentos ${ }^{2}$.

Os benzodiazepínicos (BDZ) são fármacos utilizados como hipnóticos e ansiolíticos. O primeiro composto pertencente ao grupo foi introduzido na terapia medicamentosa na década de 1960 . Na épo$\mathrm{ca}$, apresentava vantagens em relação aos barbitúricos, que provocavam inúmeros efeitos adversos ${ }^{3}$. Os efeitos farmacológicos que os $\mathrm{BDZ}$ exercem no sistema nervoso central consistem em redução da ansiedade, agressão, sedação e indução do sono, redução do tônus muscular e coordenação e efeito anticonvulsivante . $^{4}$

Os efeitos adversos dos BDZ caracterizam-se como sonolência e diminuição da função psicomotora, efeitos que parecem potencializar-se com o avanço da idade. Em idosos, o aumento da lipossolubilidade e a redução da depuração do fármaco podem resultar em maior sedaçáo e grau de comprometimento psicomotor, causando elevações das concentrações plasmáticas da droga no organismo ${ }^{5}$. Em sua totalidade, as mudanças fisiológicas relacionadas ao envelhecimento são particularmente importantes quanto à questão da acumulação dos BDZ. A sensibilidade ao fármaco apresenta relação direta com o acúmulo de $\mathrm{BDZ}$ e seus metabólitos ativos no organismo. Os idosos alcançam, então, efeitos mais prolongados e mais intensos. Por isso, observa-se com muita frequência confusão mental e desorientação em comparação aos os jovens, sintomas que interferem na qualidade de vida dos idosos, muitas vezes impossibilitando-os de realizar suas atividades diárias ${ }^{6}$.

$\mathrm{O}$ uso de $\mathrm{BDZ}$ de meia-vida longa como fármaco de escolha no tratamento de idosos tem apresentado influência no aumento do risco de quedas, maior risco de fratura de quadril e maior chance de envolvimento de idosos em acidentes de trânsito 5 .
De acordo com os critérios Beers, os BDZ de ação prolongada devem ser evitados, e, portanto, os BDZ de curta e média ação são os medicamentos mais indicados para idosos?

\section{Metodologia}

\section{Delineamento do estudo e amostra}

Este estudo é quantitativo, do tipo descritivo analítico com corte transversal. Participaram da pesquisa 100 pacientes idosos cuja prescrição continha BDZ e que recebiam o mesmo dispensado na Central de Distribuição de Medicamentos (CDM), onde são armazenadas todas as prescrições dispensadas na Atenção Primária à Saúde do município de Alfenas, localizado na região sul do estado de Minas Gerais.

\section{Coleta dos dados e considerações éticas}

Para a coleta de dados foram abordados usuários idosos que aguardavam liberação na farmácia de controlados. Assim, foram selecionados os que faziam uso contínuo de algum BDZ. De acordo com a Resolução no $466 / 2012^{8}$, os pacientes que aceitaram participar da pesquisa assinaram o Termo de Consentimento Livre Esclarecido (TCLE). O estudo foi aprovado pelo Comitê de Ética em Pesquisa (Conep) na Universidade Federal de Alfenas (UNIFAL) sob o no 1.566.473.

As características demográficas e socioeconômicas que abrangem sexo, idade, estado civil, raça, ocupação, escolaridade e renda familiar foram coletadas por meio de um questionário socioeconômico. Para analisar a qualidade de vida, o instrumento genérico Medical Outcomes Study 36 - Item Short-Form Health Survey (SF-36), foi utilizado. O SF-36 foi traduzido e validado para o português por Ciconelli ${ }^{9}$, e pode ser utilizado em qualquer situaçáo de agravo à saúde, pois é um instrumento de verificação de característica genérica. Esse instrumento é composto por 36 itens, agregados em oito dimensóes, ou domínios, que investiga aspectos distintos: Capacidade funcional, aspectos físicos, dor, estado geral de saúde, vitalidade, aspectos sociais, aspectos emocionais e saúde mental, e prevê escores de 0 a 100 para cada dimensão ${ }^{10}$.

\section{Análise dos dados}

Para análise dos resultados obtidos após a aplicação do questionário de qualidade de vida (SF-36) e para a diferença de médias, foi utilizado o teste $T$ de Student, 
e foi considerado $5 \%$ de significância. Os valores de $\mathrm{p}$ menores que 0,05 indicam que existe diferença significativa entre as médias, e os valores de $\mathrm{p}$ maiores que 0,05 indicam que as médias sáo iguais. $\mathrm{O}$ software utilizado foi o Statistical Package for Social Sciences (SPSS) 22.011.

\section{Resultados}

Foram avaliadas as condições sociodemográficas e de qualidade de vida de 100 usuários de BDZ da atenção primária à saúde de Alfenas (MG).

Gráfico 1: Valores obtidos para os fármacos BDZ mais prescritos aos idosos entrevistados $(n=100)$.

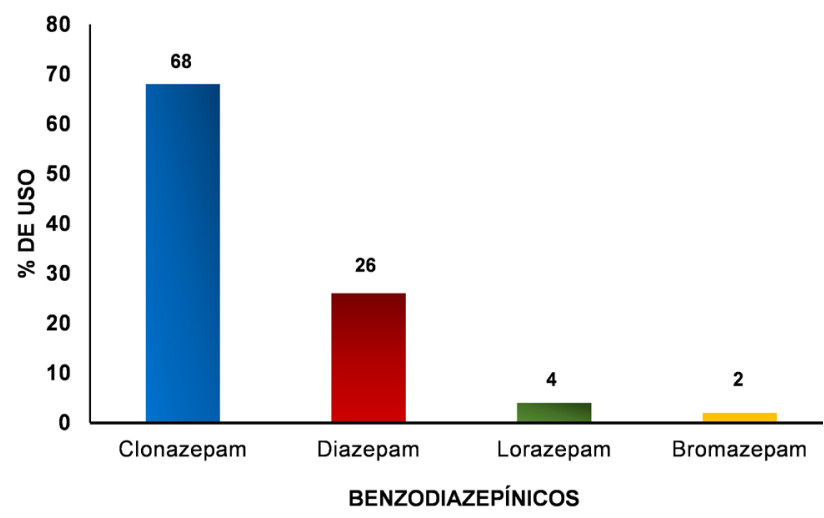

A Tabela 1 apresenta distribuição dos pacientes conforme características sociodemográficas, mostrando que 71 pacientes $(71 \%)$ eram do sexo feminino.

Tabela 1: Caracterização sociodemográfica dos usuários de BDZ entrevistados na CDM de Alfenas (MG) $(n=100)$.

\begin{tabular}{lcc} 
Variáveis & n & $\%$ \\
Gênero & 71 & 71 \\
Feminino & 29 & 29 \\
Masculino & & \\
Idade & 64 & 64 \\
60 até 70 & 36 & 36 \\
71 até 85 & & \\
Estado civil & 11 & 11 \\
Solteiro & 52 & 52 \\
Casado & 13 & 13 \\
Separado & 24 & 24 \\
Viúvo & & \\
Raça & 86 & 86 \\
Branco & 14 & 14 \\
Não branca & & continua... \\
\hline & & \\
& &
\end{tabular}

Tabela 1: Continuação.

\begin{tabular}{lcc} 
Variáveis & n & $\%$ \\
\hline Ocupaçáo & 4 & 4 \\
Afastado por doença & 69 & 69 \\
Aposentado & 1 & 1 \\
Autônomo & 2 & 2 \\
Desempregado & & \\
Grau de escolaridade & 4 & 4 \\
Analfabeto & 8 & 8 \\
Sabe ler e escrever & 31 & 31 \\
$1^{\text {o }}$ grau incompleto & 44 & 44 \\
$1^{\circ}$ grau completo & 13 & 13 \\
A partir do 2o grau incompleto & & \\
Renda & 8 & 8 \\
Até 1 salário & 85 & 85 \\
1 a 2 salários & 7 & 7 \\
\hline Acima de 2 salários & & \\
\hline
\end{tabular}

Em relação à qualidade de vida, a Tabela 2 mostra que os domínios do SF-36 apresentaram valor menor que 70. O aspecto social apresentou o maior valor: 63,9. O domínio mais comprometido foi o aspecto físico, apresentando o domínio de menor valor: 44,6.

Tabela 2: Qualidade de vida em pacientes usuários de BDZ na atenção primária à saúde do município de Alfenas, através do SF-36* $(n=100)$.

\begin{tabular}{lcc} 
Domínio & N & $\begin{array}{c}\text { Média } \\
\text { Desvio Padrão) }\end{array}$ \\
\hline Capacidade funcional & 100 & $56,7( \pm 31,4)$ \\
\hline Aspecto físico & 100 & $46,6( \pm 41,7)$ \\
Estado geral & 100 & $50,9( \pm 22,6)$ \\
\hline Dor & 100 & $50,1( \pm 28,9)$ \\
\hline Vitalidade & 100 & $54,8( \pm 22,4)$ \\
\hline Aspecto social & 100 & $63,9( \pm 29,8)$ \\
\hline Aspecto emocional & 100 & $52,0( \pm 44,5)$ \\
\hline Saúde mental & 100 & $55,8( \pm 23,6)$ \\
\hline
\end{tabular}

*The Medical Outcomes Study 36-Item Short-Form Health Survey.

A Tabela 3 mostra que os domínios que obtiveram maiores valores em idosos do sexo masculino foram capacidade funcional e aspectos sociais. Nos idosos do sexo feminino, os maiores valores foram encontrados em aspectos sociais, seguidos de saúde mental e vitalidade. Comparando a QV entre os gêneros, observa-se que a maioria dos domínios do sexo feminino apresentara valores inferiores aos do sexo masculino. São eles capacidade funcional, aspectos físicos, dor, aspectos emocionais e saúde mental. 
Tabela 3: Valores obtidos para cada domínio do SF-36 comparando os gêneros $(n=100)$.

\begin{tabular}{llll} 
Domínio & Masculino & Feminino & $\begin{array}{c}\text { Valor } \\
\text { de p }\end{array}$ \\
\hline Capacidade Funcional & $62,2( \pm 32,9)$ & $54,4( \pm 30,8)$ & 0,27 \\
\hline Aspecto Físico & $46,6( \pm 42,1)$ & $44,4( \pm 41,9)$ & 0,81 \\
\hline Estado geral & $48,4( \pm 18,7)$ & $51,9( \pm 24,1)$ & 0,44 \\
\hline Dor & $54,3( \pm 26,3)$ & $48,4( \pm 29,9)$ & 0,33 \\
\hline Vitalidade & $53,9( \pm 20,2)$ & $55,2( \pm 23,3)$ & 0,78 \\
\hline Aspecto Social & $61,2( \pm 29,8)$ & $65,0( \pm 30,0)$ & 0,57 \\
\hline Aspecto emocional & $57,5( \pm 42,6)$ & $48,9( \pm 45,4)$ & 0,42 \\
\hline Saúde Mental & $56,7( \pm 20,6)$ & $55,5( \pm 24,8)$ & 0,80 \\
\hline
\end{tabular}

Dados expressos em média \pm desvio padrão; diferença estatisticamente significativa $(\mathrm{p}<0,05)$.

Comparando as QV obtidas para o Diazepam com os dos outros BDZ (Clonazepam, Bromazepam e Lorazepam), verificou-se que em três domínios de usuários de Diazepam houve declínio na QV: Aspectos físicos, vitalidade e aspectos sociais (Tabela 4).

Nenhuma das variáveis analisadas mostrou relevância estatística para os grupos estudados.

Tabela 4: Valores obtidos para cada domínio do SF-36 comparando Diazepam com outros BDZ* $(n=100)$.

\begin{tabular}{llll} 
Domínio & Diazepam & Outros BDZ & $\begin{array}{l}\text { Valor } \\
\text { de p }\end{array}$ \\
\hline Capacidade Funcional & $55,0( \pm 29,6)$ & $52,7( \pm 32,0)$ & 0,75 \\
\hline Aspecto Físico & $45,2( \pm 43,0)$ & $44,9( \pm 41,3)$ & 0,98 \\
\hline Estado geral & $53,1( \pm 32,0)$ & $49,1( \pm 27,8)$ & 0,58 \\
\hline Dor & $51,3( \pm 26,0)$ & $50,7( \pm 21,3)$ & 0,91 \\
\hline Vitalidade & $58,8( \pm 25,3)$ & $53,4( \pm 21,1)$ & 0,33 \\
\hline Aspecto Social & $58,2( \pm 28,0)$ & $65,9( \pm 30,2)$ & 0,24 \\
\hline Aspecto emocional & $52,6( \pm 43,4)$ & $53,6( \pm 42,7)$ & 0,92 \\
\hline Saúde Mental & $53,5( \pm 26,7)$ & $56,6( \pm 22,4)$ & 0,60 \\
\hline
\end{tabular}

Dados expressos em média \pm desvio padrão; diferença estatisticamente significativa $(\mathrm{p}<0,05)$.

${ }^{*}$ Clonazepam, Lorazepam, Bromazepam.

\section{Discussão}

Este estudo foi realizado com o objetivo de avaliar a qualidade de vida de idosos usuários da atençáo primária à saúde, que fazem uso de BDZ. Verificou-se que há um comprometimento geral na qualidade de vida dos pacientes.

Dos participantes do estudo, a maioria era composta por brancos $(86 \%)$, sendo verificado baixo número de usuários negros. Esse baixo número de usuários negros pode estar relacionado a questóes históricas, culturais e sociais. Em estudos anteriores feitos por Olfson et al. ${ }^{12}$, realizado por 10 anos nos Estados Unidos, constatou-se menores índices de tratamento de depressão entre negros e hispânicos em comparação a população branca. Esses achados corroboram o resultado demonstrado neste trabalho.

Quanto aos dados demográficos e socioeconômicos dos idosos, a descrição da amostra de entrevistados encontra-se na Tabela 1.

A maior utilização de $\mathrm{BDZ}$ por idosas está de acordo com estudo anterior que relata que, de todas as características dos usuários de $\mathrm{BDZ}$, a mais evidente é ser do sexo feminino ${ }^{3,13,14}$.

Portanto, pode-se caracterizar o idoso usuário da atenção primária à saúde do município de Alfenas, que consome BDZ como: Mulher, faixa etária entre $60 \mathrm{e}$ 70 anos, baixa escolaridade (primeiro grau completo), casada e aposentada.

No Brasil, pessoas idosas são consumidoras frequentes de BZD, como evidenciam estudos epidemiológicos desenvolvidos em diferentes populaçóes, sejam elas residentes em comunidade, com prevalência estimada de $22 \%$, ou usuárias de serviços de saúde, cuja prevalência chega a $30 \%{ }^{15}$.

Apesar dos benefícios gerados pelo uso dessa classe de fármacos, como redução da ansiedade e da insônia uma revisão sobre efetividade clínica, custo/benefício e diretrizes concluiu que as evidências disponíveis sugerem maiores chances de eventos cognitivos e psicomotores adversos entre os usuários crônicos de $\mathrm{BZD}$, tais como quedas e fraturas ${ }^{15}$.

Os achados desta investigação acerca do tipo de BZD mais consumidos pelos idosos foram: 68\% utilizavam Clonazepam; 24\% utilizava o Diazepam, os fármacos Lorazepam e Bromazepam tiveram consumo mínimo.

Esses dados estão de acordo com o perfil do consumo nacional. O Boletim de Farmacoepidemiologia do Sistema Nacional de Gerenciamento de Produtos Controlados relata que no período de 2007 a 2010 o Clonazepam foi o BDZ mais utilizado no Brasil. Além disso, estudos recentes confirmam maior consumo de Clonazepam, e queda no uso do Diazepam nos anos seguintes ${ }^{14}$, dados estes similares aos resultados obtidos nesta pesquisa. Esses dados estão em desacordo com Telles Filho et al. ${ }^{16}$, que, estudando a utilização de BDZ por idosos de uma estratégia de saúde da família, verificaram que o medicamento de maior uso foi o Diazepam, seguido por Clonazepam.

De acordo com Naloto et al. ${ }^{17}$, o Clonazepam é o BZD mais adequadamente prescrito para idosos. De fato, em relação ao tipo de BZD, o Clonazepam é de ação intermediária, sendo assim, melhor para consumo quando comparado ao Diazepam, que é de longa ação e não recomendado para idosos. Isso se dá pelo fato de que o Diazepam demora mais tempo para ser 
eliminado do organismo, e por estar associado à alteraçóes decorrentes do processo de envelhecimento, podendo tornar-se fator de risco para os efeitos adversos. Entretanto, a Associaçáo Americana de Psiquiatria sugere que o Lorazepam e o Oxazepam, que não possuem metabólitos, sáo mais indicados ${ }^{18}$.

A utilização de instrumentos, como o questionário de qualidade de vida (SF-36) gera informaçóes importantes para atençáo à saúde do idoso, pois esse conceito serve como indicador no julgamento de doenças específicas e avalia o impacto físico e psicossocial que as enfermidades podem acarretar para as pessoas acometidas, permitindo melhor conhecimento acerca do paciente e de sua adaptação à condição de estar doente ${ }^{19}$.

Este estudo observou que os idosos usuários de BDZ apresentaram notável comprometimento geral da qualidade de vida. Todos os domínios apresentaram valores abaixo de 70, principalmente nos aspectos físicos e dor; o domínio que apresentou maior valor foi o aspecto social. Comparando os valores médios dos domínios do SF-36 desse trabalho com outras populaçóes idosas não usuárias dessa classe de medicamentos, verificou-se que os usuários de BZD apresentaram valores menores em cinco dos oito domínios avaliados, como aspectos físicos, dor, vitalidade, aspectos sociais e saúde mental ${ }^{20-22}$. Esse resultado sugere maior comprometimento na qualidade de vida do usuário dessa classe de medicamentos.

Quando se comparou os valores médios dos domínios do SF-36 da população idosa em relação ao gênero, notou-se que não houve diferença significante nos domínios entre os grupos, pois estes apresentaram qualidade de vida semelhante. Entretanto, observou-se que indivíduos do sexo feminino apresentaram escores ligeiramente inferiores aos do sexo masculino nos domínios capacidade funcional, dor, aspectos físicos, aspectos emocionais e saúde mental. Ressalta-se que as mulheres apresentaram tendência a considerar sua saúde pior, quando comparadas aos homens da mesma faixa etária, aspectos que também foram relatados em outros estudos ${ }^{20}$. Resultados semelhantes foram observados em pesquisas de nível de qualidade de vida para gerar diagrama populacional, e evidenciou-se que homens obtêm maior escore do que mulheres na maioria dos domínios do SF-26 23 .

Nesta pesquisa, o Diazepam, que é um medicamento potencialmente inapropriado para idosos ${ }^{5}$ foi o segundo BZD mais utilizado, mostrando que houve um número expressivo de idosos fazendo uso deste fármaco. $\mathrm{O}$ uso do Diazepam em pacientes idosos gera maior preocupaçáo, em decorrência de suas características, como alta lipossolubilidade, volume de distribuição aumentado, formação de metabólitos ativos, que levam a maiores efeitos colaterais, risco de queda e fratura. A qualidade de vida dos idosos que faziam uso do Diazepam foi comparada com outros tipos de BZD dispensados pelo SUS. Os resultados mostraram que não houve diferença significativa em nenhum domínio do SF-36.

Um dos aspectos importantes a se considerar é que existe uma linha tênue entre risco e benefício, ou seja, a elevada utilização de medicamentos pode afetar a qualidade de vida do idoso. Por outro lado, são os mesmos que, em sua maioria, ajudam a prolongar sua vida. Portanto, o uso racional dos BZD pode melhorar a qualidade de vida dos idosos. Nordon e Hubner ${ }^{24}$ relatam que a maioria dos problemas de origem psicológica ou psicossocial é vista primariamente pelo clínico geral, no atendimento primário, ressaltando que, se a prescrição do BZD é iniciada nesse momento, e erroneamente, pode conduzir a um círculo vicioso com chances de durar vários anos.

Como limitaçóes principais deste estudo destaca-se que a utilização de um instrumento genérico de qualidade de vida pode não avaliar aspectos mais específicos da qualidade de vida na terceira idade. A amostragem por demanda de serviço em um centro de referência pode não refletir rigorosamente todo o universo de idosos residentes na comunidade.

\section{Conclusão}

Os achados deste estudo evidenciam que o BDZ mais prescrito foi o Clonazepam. Entretanto, o Diazepam ainda continua com uma taxa elevada de consumo, sendo o segundo tipo mais utilizado.

Há um comprometimento geral na qualidade de vida dos idosos usuários de BDZ, residentes no município de Alfenas (MG). Os domínios mais afetados foram os aspectos físicos e a dor. Não houve alteração da qualidade de vida quando comparados os diferentes tipos de BDZ utilizados, tampouco quando comparados a outros gêneros de BDZ.

Sugere-se que sejam realizados mais estudos buscando identificar a qualidade de vida de idosos usuários de $\mathrm{BZD}$, com um número maior de participantes. Isso pode permitir a realização de melhores açóes para promoção do envelhecimento saudável e promoção do uso racional de medicamentos na atenção primária à saúde do município.

\section{Referências}

1. Mosegui GBG, Rozenfeld S, Veras RPV, Vianna CMM. Avaliação da qualidade do uso de medicamentos em idosos. Rev Saúde Públ. 1999;33(5):437-44.

2. Loyola Filho AI, Uchoa E, Firmo JOA, Lima-Costa MF. Estudo de base populacional sobre o consumo de medicamentos entre idosos: Projeto Bambuí. Cad. Saúde Pública. 2005;21(2):545-53. 
3. Cruz AV, Fulone I, Alcalá M, Fernandes AA, Montebelo MI, Lopes LC. Uso crônico de diazepam em idosos atendidos na rede pública em Tatuí-SP. Rev Ciênc Farm Básica Apl. 2006;27(3):259-67.

4. Rang HP, Dale MM, Ritter JM, Flower RJ, Henderson G. Farmacologia. $7^{\mathrm{a}}$ ed. Rio de Janeiro: Elsevier; 2012.

5. Baldoni AO, Chequer FMD, Ferraz ERA, Oliveira DP, Pereira LRL, Dorta DJ. Eldery and drugs: risks and necessity of rational use. Braz J Pharm Sci. 2010;46(4):617-32.

6. Griffin CE III, Kaye AM, Bueno FR, Kaye AD. Benzodiazepinepharmacology and central nervous system-mediated effects. Ochsner J. 2013;13(2):214-23.

7. Fick DM, Cooper JW, Wade WE, Waller JL, Maclean JR, Beers MH. Updating the Beers criteria for potentially inappropriate medication use in older adults: results of a US consensus panel of experts. Arch Intern Med. 2003;163(22):2716-24.

8. Brasil. Ministério da Saúde. Fundação Nacional de Saúde. Resolução no 466, de 12 de dezembro de 2012, sobre pesquisas envolvendo seres humanos. Brasília, DF: Ministério da Saúde; 2013.

9. Ciconelli RM. Tradução para o português e validação do questionário genérico de avaliação de qualidade de vida medical outcomes study 36 - item short -form health survey (SF-36). São Paulo. Tese [Doutorado em Ciências da Saúde Aplicadas à Reumatologia] - Escola Paulista de Medicina de São Paulo da Universidade Federal de São Paulo; 1997.

10. Soares DA, Toledo JAS, Santos LF, Lima RMB, Galdeano LE. Qualidade de vida de portadores de insuficiência cardíaca. Acta Paul Enferm. 2008;21(2):243-48.

11. IBM Corp. Released 2013. IBM SPSS Statistics for Windows, Version 22.0. Armonk, NY: IBM Corp; 2013.

12. Olfson M, Marcus SC, Druss B, Elinson L, Tanelian T, Pincus HA. National trends in the outpatient treatment of depression. JAMA. 2014;287(2):203-9.

13. Alvarenga JM, Giacomin KC, Loyola Filho AI, Uchôa E, Firmo JOA. Uso crônico de benzodiazepínicos entre idosos. Rev Saúde Pública. 2014;48(6):866-72.
14. Azevedo AJP, Araújo AA, Ferreira MAF. Consumo de ansiolíticos benzodiazepínicos: uma correlação entre dados do SNGPC e indicadores sociodemográficos nas capitais brasileiras. Ciênc Saúde coletiva. 2016;21(1):83-90.

15. Alvarenga JM, Loyola Filho AI, Giacomin KC, Uchoa E, Firmo AO. Uso de benzodiazepínicos entre idosos: o alívio de “jogar água no fogo", para não pensar e dormir. Rev Bras Geriatr Gerontol. 2015;18(2):249-58.

16. Telles Filho PCP, Chagas AR, Pinheiro MLP, Lima AMJ, Durão AMS. Utilização de benzodiazepínicos por idosos de uma estratégia de saúde da família: implicaçóes para a enfermagem. Esc Anna Nery Rev Enferm. 2011;15(3):581-86.

17. Naloto DCC. Prescrição de benzodiazepínicos para adultos e idosos de um ambulatório de saúde mental. Ciênc Saúde Coletiva. 2016;21(4):1267-76.

18. McIntosh B, Clark M, Spry C. Benzodiazepines in older adults: a review of clinical effectiveness, cost-effectiveness, and guidelines. [Internet]. Ottawa (ON): Canadian Agency for Drugs and Technologies in Health; 2011 jan. [acesso em 2017 jun. 13]. Disponível em: https://goo.gl/RXS5ID.

19. Carvalho MV, Siqueira LB, Sousa ALL, Jardim PCBV. The influence of hypertension on quality of life. Arq Bras Cardiol. 2013;100(2):164-74.

20. Pimenta FAP, et al. Avaliação da qualidade de vida de aposentados com a utilizaçáo do questionário SF-36. Rev Assoc Med Bras. (1992). 2008;54(1):55-60.

21. Campolina AG, Dini PS, Ciconelli RM. Impacto da doença crônica na qualidade de vida de idosos da comunidade em São Paulo (SP, Brasil). Ciênc Saúde Coletiva. 2011;16(6):2919-25.

22. Coelho ESP, Pedroso MFBP. Avaliação da qualidade de vida em idosos residentes no município de Santos/SP. Rev Ceciliana. 2012;4(1):4-8.

23. Santos, PR. Relação do sexo e da idade com nível de qualidade de vida em renais crônicos hemodialisados. Rev Assoc Med Bras. (1992). 2006;52(5):356-59.

24. Nordon DG, Hübner CVK. Prescrição de benzodiazepínicos por clínicos gerais. Diagn Tratamento. 2009;14(2):66-9.

\section{Como citar este artigo:}

Souto SMT, Podestá MHMC, Souza WA, Almeida GG. Qualidade de vida de idosos usuários de benzodiazepínicos. Rev. Aten. Saúde. 2017;15(52):96-101. 